\title{
Calculations of neoclassical viscous damping on flux surfaces near magnetic islands in the Helically Symmetric Experiment
}

\author{
S. P. Gerhardt, ${ }^{\text {a) }}$ D. T. Anderson, and J. N. Talmadge \\ HSX Plasma Laboratory, University of Wisconsin-Madison, Madison, Wisconsin 53703
}

(Received 9 August 2004; accepted 4 October 2004; published online 14 December 2004)

\begin{abstract}
Neoclassical viscous damping rates have been calculated for the helically symmetric experiment [F. S. B. Anderson et al., Fusion Technol. 27, 273 (1995)], concentrating on the effects of magnetic surface shape changes due to magnetic islands. The interaction between the main helical spectral component and magnetic surface shape changes caused by an island chain introduces symmetry breaking spectral components on closed surfaces near the island chain. Results indicate that small islands present in the quasihelically symmetric configuration can give rise to an observable but not problematic deviation from quasisymmetry on surfaces near the islands. These islands can be eliminated via a small reduction in the rotational transform. Large islands can be produced by the introduction of the $t=4 / 4$ resonance inside the last closed magnetic surface. These islands result in not only a significant reduction in the volume of closed nested magnetic surfaces, but also an elevated level of viscous damping on magnetic surfaces adjacent to the islands. () 2005 American Institute of Physics. [DOI: 10.1063/1.1825390]
\end{abstract}

\section{INTRODUCTION}

The Helically Symmetric Experiment (HSX) ${ }^{1}$ is a member of the family of advanced quasisymmetric stellarators. These stellarators are defined by the property that the Fourier decomposition of the magnetic field strength in magnetic coordinates is dominated by a single harmonic. In the case of quasihelical symmetry (QHS) ${ }^{2}$ in HSX, the single large spectral component has toroidal $(n)$ and poloidal $(m)$ mode numbers $(n, m)=(4,1)$. The $(n, m)=(0,1)$ toroidal curvature component of the magnetic field spectrum is reduced to the level of an aspect ratio 400 stellarator, even though the physical aspect ratio of HSX is $\sim 10$. In this sense, HSX has the transport properties of a straight stellarator, but in toroidal geometry.

In a conventional stellarator without a direction of symmetry, the three dimensional nature of the magnetic field leads to poor neoclassical transport properties. These include a diffusion coefficient scaling such as the inverse collision frequency ${ }^{3}$ and large viscous damping of plasma flows in all directions on a flux surface. ${ }^{4}$ A quasisymmetric stellarator, on the other hand, is able to largely eliminate these poor transport properties. In general, these configurations have neoclassical transport properties similar to an axisymmetric configuration such as a tokamak.

It is of interest to know what effects can break the quasisymmetry, as any large deviation from quasisymmetry can cause the neoclassical transport to revert to the level of a conventional stellarator. One mechanism for the breaking of quasisymmetry lies in the formation of magnetic islands. On surfaces near the magnetic island, significant changes can occur in the shape of the flux surfaces even through the contours of constant $|B|$ may not be significantly different than they would have been in the absence of the resonant error

\footnotetext{
${ }^{a)}$ Present address: Princeton Plasma Physics Laboratory, Princeton, New Jersey 08543.
}

field which lead to the island. Field lines on these surfaces near islands will traverse through the magnetic field on different trajectories than they would have in the absence of the island. The variation of $|B|$ along the field line will thus be different than the case without the island, causing a deviation from the ideal quasisymmetric properties of the configuration. This transport mechanism has been noted in the context of magnetic islands in otherwise axisymmetric tokamaks. ${ }^{5}$

In general, efforts are made during the stellarator design process to avoid the presence of large island chains. In HSX and many other low shear stellarators, the rotational transform profile is chosen such that there are no low-order resonances; $t$ varies from 1.05 on axis to 1.12 at the last closed magnetic surface (LCMS) for the base configuration of HSX. Other stellarators with low-order rational surfaces present have relied on large magnetic shear to minimize the island width. More recently, island elimination techniques ${ }^{6}$ have been applied to the design of the National Compact Stellarator Experiment (NCSX). ${ }^{7}$ Nevertheless, residual small island chains may exist; the $t=12 / 11$ resonance in the base configuration of HSX is an example. A computational study of the effects of the surface deformations associated with the islands is the purpose of this paper. In particular, we examine the modifications to the Hamada spectrum on surfaces near the islands, and then consider the impact of the islands on the neoclassical viscous damping as calculated from the Hamada spectrum.

The effects of magnetic islands on viscous damping in a stellarator have probably already been observed, in the context of access to edge transport barriers ( $H$-modes). $\quad H$-mode access in the stellarator Wendelstein 7- $\mathrm{AS}^{8}$ is limited to small windows surrounding certain values of edge rotational transform. ${ }^{9}$ These particular configurations correspond to cases where there are no large islands inside the separatrix. Calculations of the poloidal damping coefficient reveal a reduction in the viscous damping in these 
configurations. ${ }^{10}$ The hypothesis in this case is that the reduced viscous damping in these configuration windows allows poloidal shear flow ${ }^{11}$ to develop, leading in turn to turbulence suppression and the transition to $H$-mode. A similar relationship between the edge topology and $H$-mode access may have been observed in Heliotron-J. ${ }^{12}$ On the other hand, experiments in the Large Helical Device have demonstrated both the existence of radial electric field shear and the reduction of heat transport at the boundary of a magnetic island. ${ }^{13}$

HSX has unique properties for studying the viscous damping due to magnetic islands. The quasisymmetric field in the base configuration is generated with a set of 48 nonplanar coils. Each nonplanar coil has a planar auxiliary coil associated with it. These auxiliary coils can be used to add or subtract toroidal field from that produced by the main coils. When the field of all 48 auxiliary coils adds to the field of the main coils, the rotational transform and well depth are decreased and the configuration moves toward a magnetic hill; these are known as "Hill" configurations. When the field of all auxiliary coils subtracts from the main field, the rotational transform is raised and the plasma well depth is deepened; this is known as the "Well" configuration. A different configuration is formed when alternating groups of six auxiliary coils add or subtract from the toroidal field of the main coils. This leads to the "Mirror" configuration with a large $(n, m)$ $=(4,0)$ symmetry breaking component in the magnetic field spectrum. The configurations accessed through the auxiliary coils are denoted by the labels above (Hill, Well, and Mirror) and by the percentage of amp-turns in the auxiliary coil set compared to the amp turns in the main coils. HSX has a major radius of $1.2 \mathrm{~m}$ and a minor radius of from 9 to $13 \mathrm{~cm}$, depending on the configuration.

In this research, the neoclassical damping rates in HSX have been computed using the model developed by Coronado and Talmadge. ${ }^{14}$ These damping rates are calculated for four separate configurations. In the first case, the intrinsic small islands in the vacuum field of the base quasisymmetric configuration are considered. Having established this baseline, a case is considered where the rotational transform has been lowered sufficiently to exclude the islands which cause the increased damping. Next, a case is considered where the rotational transform has been lowered slightly further and the $t=4 / 4$ rational surface enters the confinement volume. This resonance leads to large symmetry breaking on the highly deformed surfaces inside and outside of the island chain. Finally, a Mirror configuration of HSX is considered which contains both $t=8 / 7$ magnetic island as well as a large $(n, m)=(4,0)$ symmetry breaking component.

The ordering of the remainder of this paper is as follows. Section II will provide some details of the calculation of the viscous damping rates and explain the physical meaning of the damping rates. Section III will provide comparisons between the four different configurations of HSX discussed in the previous paragraph. Section IV will compare the viscous damping in the four different configurations. Section V will summarize the calculation.

\section{CALCULATION OF THE VISCOUS DAMPING RATES}

To quantify the effects of magnetic islands on the damping of plasma flows, we use the model by Coronado and Talmadge. ${ }^{14}$ This model was developed to explain the spin-up or spin-down of a plasma subject to an externally driven radial current. The external current in the model may be that drawn by a biased electrode, although other nonambipolar particle transport mechanisms, such as ion orbit loss ${ }^{15}$ would be consistent with the model. The model provides two time scales for the plasma flow evolution, corresponding to two different directions on a magnetic surface. The portions of the modeling which are utilized in this paper are described briefly here.

The first order parallel and poloidal momentum balance equations in Hamada coordinates ${ }^{16}$ are given by

$$
\begin{aligned}
& m_{\mathrm{i}} N_{\mathrm{i}} \frac{\partial}{\partial \mathrm{t}}\langle\mathbf{B} \cdot \mathbf{U}\rangle=-\langle\mathbf{B} \cdot \boldsymbol{\nabla} \cdot \Pi\rangle, \\
& m_{\mathrm{i}} N_{\mathrm{i}} \frac{\partial}{\partial \mathrm{t}}\left\langle\mathbf{B}_{\mathrm{P}} \cdot \mathbf{U}\right\rangle=-\frac{\sqrt{g} B^{\zeta} B^{\alpha}}{c}\left\langle\mathbf{J}_{\text {plasma }} \cdot \boldsymbol{\nabla} \psi\right\rangle-\left\langle\mathbf{B}_{\mathrm{P}} \cdot \boldsymbol{\nabla} \cdot \Pi\right\rangle .
\end{aligned}
$$

In these expressions, $m_{\mathrm{i}}$ is the ion mass, $N_{\mathrm{i}}$ is the ion density, $\psi$ is the toroidal flux, $B$ is the magnetic field, $B_{\mathrm{P}}$ is the poloidal magnetic field, $\Pi$ is the viscous stress tensor, $\sqrt{g}$ is the Jacobian, $c$ is the speed of light, and $B^{\alpha}$ and $B^{\zeta}$ are the poloidal and toroidal contravariant components of the magnetic field. The notation $\langle\cdots\rangle$ represents a flux surface average. ${ }^{16}$ The currents flowing through the plasma and the electrode ( $J_{\text {plasma }}$ and $J_{\text {ext }}$, respectively) are related to the electric field using the radial component of Ampère's law: ${ }^{14}$

$$
\frac{\partial}{\partial \mathrm{t}} \frac{\partial \Phi}{\partial \psi}\langle\boldsymbol{\nabla} \psi \cdot \boldsymbol{\nabla} \psi\rangle=4 \pi\left(\left\langle\mathbf{J}_{\text {plasma }} \cdot \boldsymbol{\nabla} \psi\right\rangle+\left\langle\mathbf{J}_{\mathrm{ext}} \cdot \boldsymbol{\nabla} \psi\right\rangle\right) .
$$

To calculate the viscosities, we Fourier decomposed the magnetic field in the Hamada coordinate ${ }^{16}$ toroidal and poloidal angles:

$$
B=B_{o} \sum_{n, m} b_{n m} \cos (n \zeta-m \alpha) .
$$

This Fourier decomposition has been accomplished for HSX using a modification ${ }^{17}$ of the technique originally developed for the calculation of the Boozer spectra. ${ }^{18}$ The calculation of the Hamada spectrum can yield hundreds of spectral components to accurately model the magnetic field. We generally keep only those spectral components whose amplitude is greater than $10^{-4}$ times the average field on the flux surface. The inclusion of additional terms does not change the calculated damping rates. Note that we never calculate the Hamada spectrum exactly at the rational surface, but only on the nested irrational surfaces close to the rational surface.

The neoclassical viscosities in Eqs. (1) and (2) can be written in the plateau regime ${ }^{19}$ as $\langle\mathbf{B} \cdot \boldsymbol{\nabla} \cdot \Pi\rangle=\mu_{\alpha} U^{\alpha}+\mu_{\zeta} U^{\zeta}$ and $\left\langle\mathbf{B}_{\mathrm{P}} \cdot \boldsymbol{\nabla} \cdot \Pi\right\rangle=\mu_{\alpha}^{(\mathrm{P})} U^{\alpha}+\mu_{\zeta}^{(\mathrm{P})} U^{\zeta}$, where $\mu_{\alpha}=\kappa\left(B^{\alpha} \alpha_{\mathrm{P}}\right.$ 
$\left.+B^{\zeta} \alpha_{\mathrm{C}}\right), \quad \mu_{\zeta}=\kappa\left(B^{\alpha} \alpha_{\mathrm{C}}+B^{\zeta} \alpha_{\mathrm{T}}\right), \quad \mu_{\alpha}^{(\mathrm{P})}=\kappa B^{\alpha} \alpha_{\mathrm{P}}, \quad$ and $\quad \mu_{\alpha}^{(\mathrm{T})}$ $=\kappa B^{\alpha} \alpha_{\mathrm{C}}$. These expressions in turn use $\kappa=\pi^{1 / 2} P B_{\mathrm{o}} / v_{\mathrm{t}} B^{\zeta}$

$$
\begin{aligned}
& \alpha_{\mathrm{T}}=\sum_{n, m \neq 0} \frac{n^{2} b_{n, m}^{2}}{|n-m \boldsymbol{t}|}, \\
& \alpha_{\mathrm{P}}=\sum_{n, m \neq 0} \frac{m^{2} b_{n, m}^{2}}{|n-m \boldsymbol{t}|}, \\
& \alpha_{\mathrm{C}}=-\sum_{n, m \neq 0} \frac{n m b_{n, m}^{2}}{|n-m \boldsymbol{t}|} .
\end{aligned}
$$

In these expressions, $P$ the pressure and $v_{\mathrm{t}}$ the thermal velocity. These viscosities are only strictly valid for time scales longer than the ion-ion collision time, ${ }^{20}$ placing a limit on the applicability of the formula to the shortest time scales.

Using the viscosities provided above, Eq. (3), and assuming incompressibility, Eqs. (1) and (2) can be converted to a system of first order linear differential equations for the parallel flows and radial electric field. ${ }^{14}$ The external current (for instance, that in a biased electrode) appears as a source term in these differential equations. If the current is abruptly terminated, the flows and electric fields will decay with two time scales, given by

$$
\left.\begin{array}{l}
\gamma_{\mathrm{s}} \\
\gamma_{\mathrm{f}}
\end{array}\right\}=-\frac{v_{1}}{2 \Omega} \pm\left[\left(\frac{v_{1}}{2 \Omega}\right)^{2}+\frac{v_{\zeta}^{(\mathrm{P})} v_{\alpha}-v_{\alpha}^{(\mathrm{P})} v_{\zeta}}{\Omega}\right]^{1 / 2},
$$

where $I_{\mathrm{o}}=\left(B^{\alpha} / 2 \mathrm{x} c\right)^{2}\langle\nabla \psi \cdot \nabla \psi\rangle /\left(4 \mathrm{x} m_{\mathrm{i}} N_{\mathrm{i}}\left\langle\mathbf{B}_{\mathrm{P}} \cdot \mathbf{B}_{\mathrm{P}}\right\rangle\right)$ and $\Omega=1$ $+I_{\mathrm{o}}-\left(\left\langle\mathbf{B} \cdot \mathbf{B}_{\mathrm{P}}\right\rangle\right)^{2} /\left(\langle\mathbf{B} \cdot \mathbf{B}\rangle\left\langle\mathbf{B}_{\mathrm{P}} \cdot \mathbf{B}_{\mathrm{P}}\right\rangle\right)$. The viscous frequencies are defined as $\nu_{\alpha}=\mu_{\alpha} B^{\zeta} / m_{\mathrm{i}} N_{\mathrm{i}}\langle\mathbf{B} \cdot \mathbf{B}\rangle, \quad \nu_{\zeta}=\mu_{\zeta} B^{\zeta} / m_{\mathrm{i}} N_{\mathrm{i}}\langle\mathbf{B} \cdot \mathbf{B}\rangle$, $\nu_{\alpha}^{(\mathrm{P})}=\mu_{\alpha}^{(\mathrm{P})} B^{\alpha} / m_{\mathrm{i}} N_{\mathrm{i}}\left\langle\mathbf{B}_{\mathrm{P}} \cdot \mathbf{B}_{\mathrm{P}}\right\rangle$, and $\nu_{\zeta}^{(\mathrm{P})}=\mu_{\zeta}^{(\mathrm{P})} B^{\alpha} / m_{\mathrm{i}} N_{\mathrm{i}}\left\langle\mathbf{B}_{\mathrm{P}} \cdot \mathbf{B}_{\mathrm{P}}\right\rangle$, and the frequency $\nu_{1}$ is given by

$$
\begin{aligned}
v_{1}= & v_{\alpha}^{(\mathrm{P})}+\left(1+I_{\mathrm{o}}\right)\left(t v_{\alpha}+v_{\zeta}\right)-\frac{\left\langle\mathbf{B} \cdot \mathbf{B}_{\mathrm{P}}\right\rangle}{\langle\mathbf{B} \cdot \mathbf{B}\rangle}\left(v_{\alpha}^{(\mathrm{P})}+q v_{\zeta}^{(\mathrm{P})}\right) \\
& -\frac{\left\langle\mathbf{B} \cdot \mathbf{B}_{\mathrm{P}}\right\rangle}{\left\langle\mathbf{B}_{\mathrm{P}} \cdot \mathbf{B}_{\mathrm{P}}\right\rangle} t v_{\alpha} .
\end{aligned}
$$

These two rates in Eq. (6) will be referred to as the fast rate $\left(\gamma_{\mathrm{f}}\right)$ and the slow rate $\left(\gamma_{\mathrm{s}}\right)$. For example, in the case of a perfectly axisymmetric tokamak, the slow rate corresponds to damping of flows in the toroidal direction, and is equal to zero because there is no variation in $|B|$ in that direction. More generally, the slow rate corresponds to damping of flows along the approximate direction of symmetry in $|B|$ if such a direction exists, while the fast rate corresponds to damping of flows which are across the direction of symmetry and their accompanying parallel flows. ${ }^{17}$ These two damping rates, as well as the magnetic field spectrum itself, will be used as figures of merit when comparing the effects of magnetic islands in different configurations of HSX.

These calculations include only the effects of neoclassical parallel viscosity. The original paper by Coronado and Talmadge ${ }^{14}$ includes the effects of ion-neutral collisions on the damping of flows. Given that these collisions would only obscure the neoclassical effects that this paper is designed to

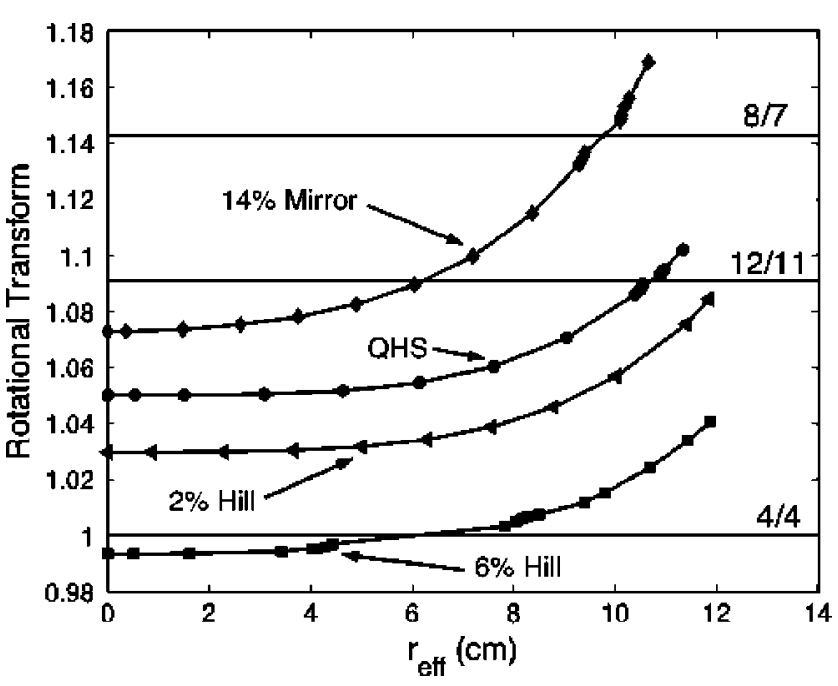

FIG. 1. Rotational transform profiles for the QHS, 2\% Hill, 6\% Hill, and $14 \%$ Mirror configurations. Important low-order resonances are displayed as horizontal lines. These symbols for the different configurations will be maintained throughout this paper.

explore, the damping due to neutrals has been ignored. The effects of any shear viscosity are also neglected.

All calculations are done using the vacuum field of HSX as calculated by a Biot-Savart code. We assume that in the low- $\beta$ plasmas typical of HSX to date, the plasma does not modify the magnetic field in any appreciable way. In particular, it is assumed that the vacuum islands still exist in the presence of the plasma, i.e., island healing ${ }^{21,22}$ does not occur.

\section{SYMMETRY BREAKING ISLAND PERTURBATION IN FOUR DIFFERENT CONFIGURATIONS OF HSX}

The calculations presented are accomplished through a number of steps. For a given configuration of HSX, detailed Poincarre plots are generated of finely spaced magnetic surfaces. These calculations are used to identify the LCMS and the location of any island chains, as well as magnetic surfaces on which to calculate the Hamada spectra. If the island chains were not precisely localized in this step, the magnetic surfaces in the vicinity of the islands are calculated with more fine resolution until the field line launching locations are determined which fill in surfaces sufficiently close to the island chains. The Hamada spectrum is then calculated on the closely spaced surfaces very near the islands and on more coarsely spaced surfaces away from the islands.

The rotational transform profiles for the four configurations studied in this paper are presented in Fig. 1. Low-order natural resonaces are also shown as horizontal lines. There is a $t=12 / 11$ surface present inside the last closed flux surface (LCFS) in the base QHS configuration. This resonance has been excluded in the $2 \%$ Hill configuration, where the auxiliary coils have been used to reduce the rotational transform. Continuing to reduce the rotational transform causes the $t=4 / 4$ surface to enter the plasma at $\approx 5.25 \%$ Hill. The rotational transform for the $6 \%$ Hill case is shown in the figure, illustrating that the $t=4 / 4$ surface exists at approxi- 

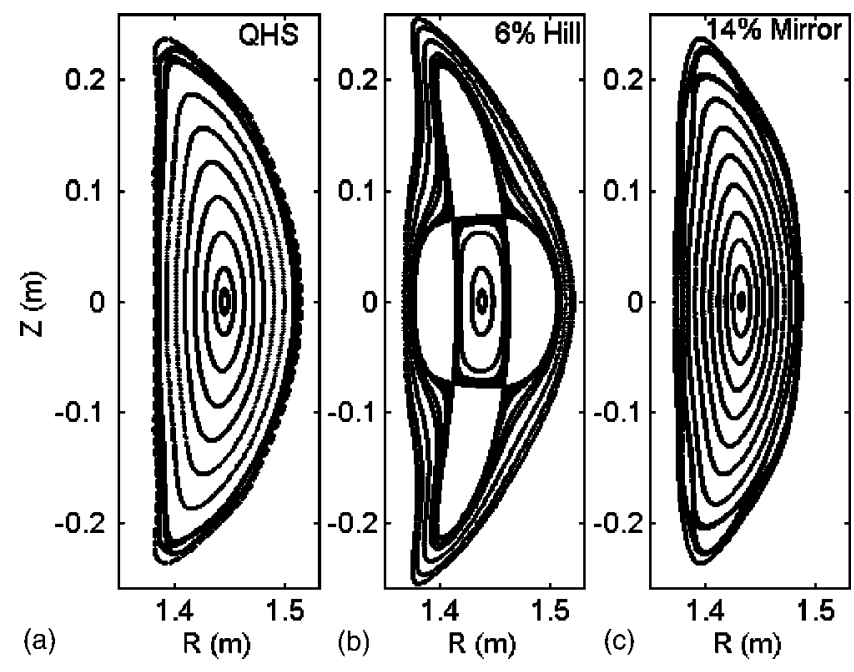

FIG. 2. Magnetic surfaces at the ellipsoidal shaped symmetry plane for the QHS, 6\% Hill, and 14\% Mirror configurations. The rational surfaces apparent in Fig. 1 appear as gaps between the closed magnetic surfaces in these plots. The surfaces displayed in the calculations are the surfaces on which the Hamada spectrum and damping rates are calculated.

mately the half radius in this configuration. Also shown is the rotational transform for the $14 \%$ Mirror configuration. As noted previously, this configuration contains a large $(n, m)$ $=(4,0)$ symmetry breaking spectral component, whose amplitude is approximately constant over the full minor radius. The rotational transform is also slight raised in this case, leading to $\mathrm{a} t=8 / 7$ resonance inside the LCMS. Note that in this and other plots, the effective minor radius $r_{\text {eff }}($ in $\mathrm{cm}$ ) is defined as $r_{\text {eff }}=100\left(\psi_{a} / x \times 0.5\right)^{1 / 2}$, where $\psi_{\alpha}$ is the toroidal flux through the boundary in $\mathrm{T}^{2}$ and the field strength is always $0.5 \mathrm{~T}$ on the magnetic axis at a toroidal angle of $\Phi$ $=0$.

The magnetic surfaces for the QHS, 6\% Hill, and 14\% Mirror configurations are shown in Fig. 2 for vertical slices at the symmetry plane with approximately elliptical surfaces. Each of the resonances noted in the previous paragraph are visible as a set of gaps between closed surfaces, inside which magnetic islands exist. The surfaces shown in the graphs are the surfaces on which Hamada spectrum and the damping calculations have been performed. The surfaces are carefully chosen so that regions of the plasma with fine structure are fully resolved.

The large $t=4 / 4$ island chain in the center frame of Fig. 2 illustrates most clearly the means by which the island chains break symmetry. Near the axis, the surfaces have an elliptical shape which is similar to the surface shape in the QHS configuration. The closed surfaces near the inner separatrix of the island chain have developed a distinctly square shape. The closed surfaces beyond the outer separatrix of the island chain dive in toward the $X$ points. Moving toward the LCMS, the surfaces once again have a shape that is similar to the outer surface shape in the QHS configuration. Recall that these large deviations from the QHS surface shape are the result of a magnetic field perturbation at the level of $6 \%$ of the amp-turns of the main coil set. The volume inside various surfaces of this configuration has been calculated using a

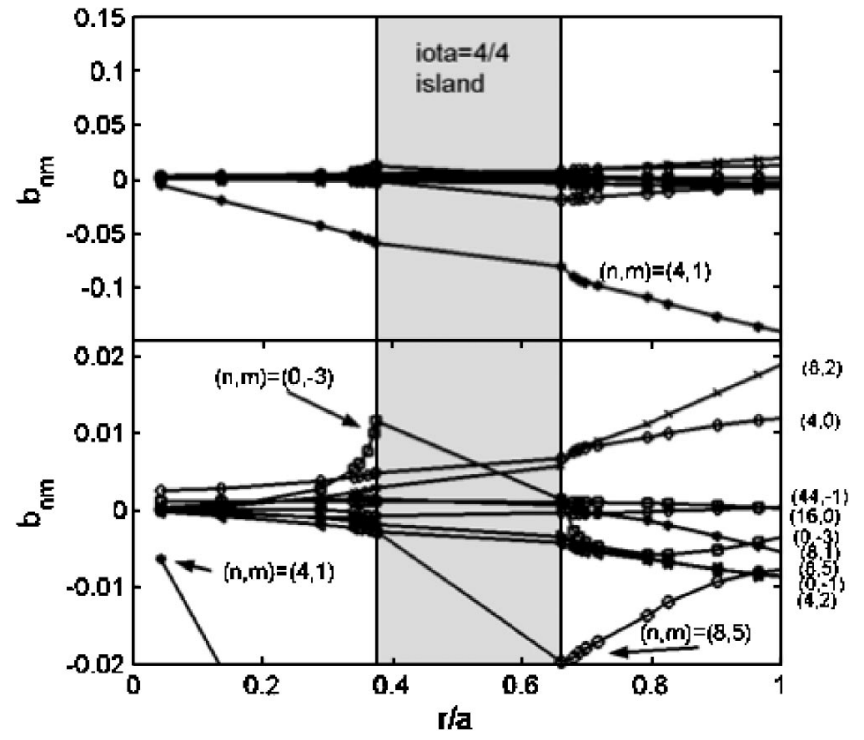

FIG. 3. (a) The complete Hamada spectrum for the $6 \%$ Hill configuration and (b) a detailed view of the smaller spectral components. The $t=4 / 4$ island chain exists in the shaded region and the spectral components are discontinuous across the gap due to the different magnetic surface shapes on either side of the island. The ten largest spectral components at $r / a=0.372$ are illustrated in the plot.

Monte Carlo integration method. The volume contained by the LCMS for this $6 \%$ Hill configuration is $\approx 0.4 \mathrm{~m}^{3}$. The volume enclosed by the last square surface inside the island separatrix is $\approx 0.055 \mathrm{~m}^{3}$, while the volume of the first closed surface beyond the island chain is $\approx 0.22 \mathrm{~m}^{3}$. Hence, this island chain contains $\approx 40 \%$ of the volume within the LCMS.

The Hamada spectrum for the $6 \%$ Hill configuration is shown in Fig. 3. The top frame of the figure illustrates the entire spectrum. Note that the $(n, m)=(4,1)$ spectral component is the largest spectral component, even on surfaces near the magnetic island. The bottom frame shows the same data in a view that has been zoomed in to illustrate the small spectral components. The components shown are the largest ten for the magnetic surface at $r / a=0.373$, i.e., just before the inner separatrix of the island chain. As the surface shape transitions from elliptical to square, there is a distinct increase in the number and amplitude of symmetry breaking components. Crossing the island chain yields a discontinuity in the spectral components due to the very different surface shape. Approaching the LCMS yields a decrease in some spectral components associated with the perturbed surface shape [the $(n, m)=(8,5)$ or $(0,-3)$ spectral components, for instance], and an increase in those spectral components which grow towards the edge in the QHS configuration [the $(n, m)=(8,2)$ harmonic of the main spectral component, for instance].

The symmetry breaking spectral components are determined by the interaction of the main helical spectral component and the island perturbation. In the $6 \%$ Hill case, for instance, the $(n, m)=(4,1)$ perturbation mixes with the $(4,4)$ perturbation, leading to large spectral components with mode numbers $(n, m)=(4-4,1-4)=(0,-3)$ and $(n, m)=(4+4,1$ $+4)=(8,5)$. Smaller spectral components not shown in the 


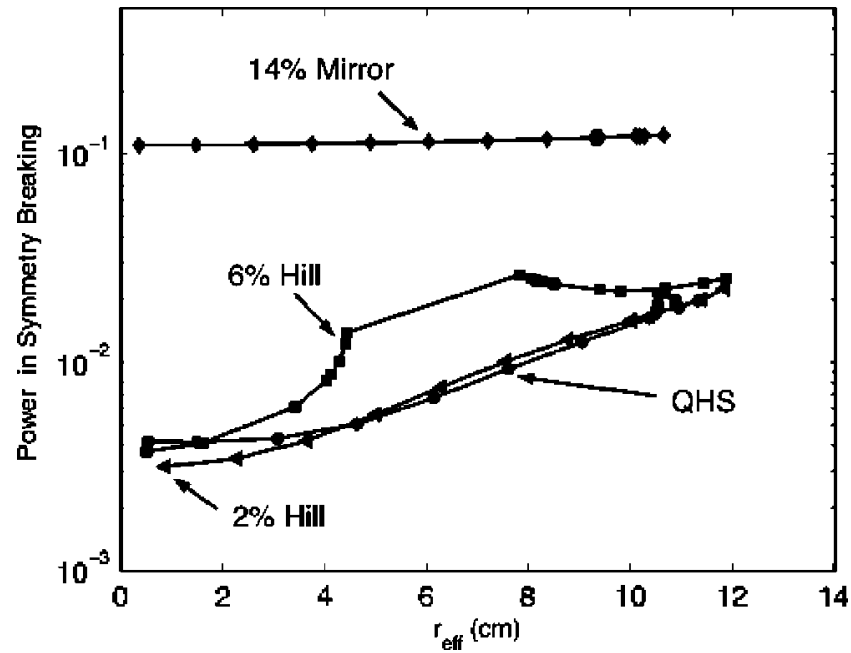

FIG. 4. The fractional power in symmetry breaking spectral components for the four configurations, where the power is defined using Eq. (8).

figure are those based on the second, third, and fourth harmonics of the field perturbation, i.e., $(n, m)=(4,1)+2(4,4)$ $=(12,9),(n, m)=(4,1)-2(4,4)=(-4,-7)$, etc. Similarly, the largest perturbations associated with the $t=12 / 11$ island chain in the QHS configuration are the $(n, m)$ $=(4,1) \pm(12,11)=(16,12)$ and $(-8,-10)$ spectral components.

The planar auxiliary coils add some toroidal curvature to the magnetic field spectrum. Note, though, that this $(n, m)$ $=(0,-1)$ spectral component is still small compared to many other components in the spectrum. We thus infer that any increase in viscous damping is due to the modification of the surface shape, not simply the addition of a small amount of toroidal curvature.

As a final assessment of the complexity of the Hamada spectrum, it is useful to plot the total power in symmetry breaking spectral components for the different configurations. This quantity is defined as

$$
\text { Power }=\left(\sum_{\substack{(n, m) \neq(0,0),(4,1) \\ n / 4 \neq m}} b_{n, m}^{2}\right)^{1 / 2} \text {. }
$$

Note that this definition excludes the average field on a flux surface $\left(b_{0,0}\right)$, the main helical spectral component $\left(b_{4,1}\right)$, and all spectral components with the same helicity as the $(n, m)$ $=(4,1)$ component. This figure of merit is shown in Fig. 4 for the four configurations of interest.

The power in symmetry breaking components in the QHS configuration shows a general rise toward the edge, with a final level of about $2 \%$ at the LCMS. There is an increase in symmetry breaking power in the vicinity of the $t=12 / 11$ island, but it never exceeds the level at the LCMS. The 2\% Hill case, where the $t=12 / 11$ island has been excluded, shows simply a monotonic rise of the symmetry breaking power toward the edge. The 14\% Mirror case shows large symmetry breaking across the entire plasma cross section. This is due to the large $(n, m)=(4,0)$ spectral component introduced by the auxiliary coils. The effects of

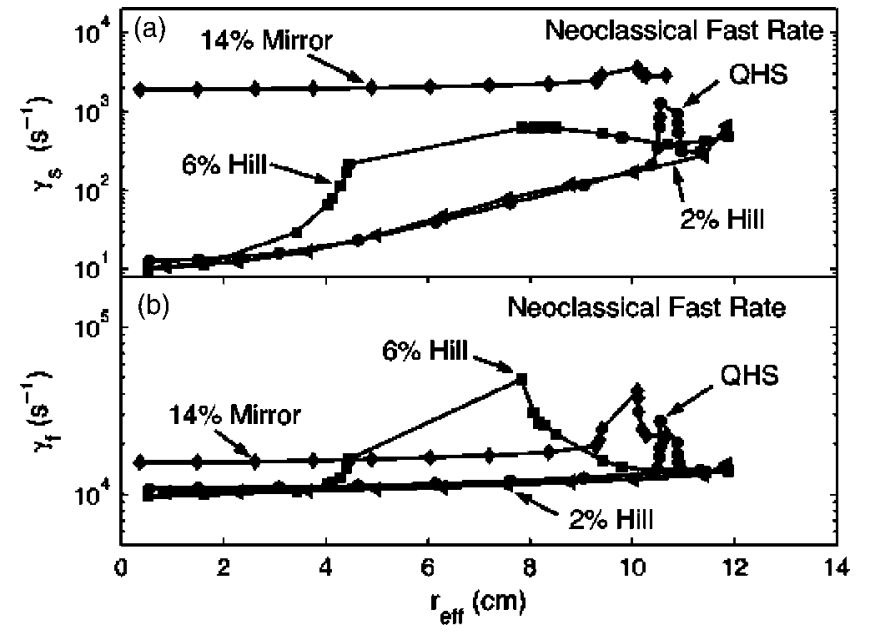

FIG. 5. (a) The fast and (b) slow damping rates, as defined using Eq. (6). The magnetic islands cause local increases in the damping rates, while there is an increase in viscous damping across the entire minor radius in the mirror configurations.

the $t=8 / 7$ island chain are not visible in this plot, as they are obscured by the symmetry breaking of the large $(n, m)$ $=(4,0)$ spectral component. Finally, the symmetry breaking impact of the $t=4 / 4$ island chain in the $6 \%$ Hill configuration is clearly visible on either side of the island chain. Note that the QHS, 2\% Hill, and 6\% Hill configurations have very similar power in symmetry breaking spectral components at the LCMS, which is free of large scale island deformation in all three cases.

\section{VISCOUS DAMPING RATES IN FOUR CONFIGURATIONS OF HSX}

Having calculated the Hamada spectra, it is possible to determine the viscous damping rates as described in Sec. II. The plasma parameters are assumed to be the same in all calculations. A flat ion temperature profile with $T_{\mathrm{i}}=20 \mathrm{eV}$ is used. A parabolic density profile is used with a small dc offset to provide some density at the edge. The profile is constrained to have a line-averaged density of 1 $\times 10^{12} \mathrm{~cm}^{-3}$. These conditions place the plasma in the plateau regime, where the damping rates are independent of the plasma density. Given the flat ion temperature profile used in the calculations, the profile shapes of the damping rates are due to the magnetic geometry only.

The slow damping rates for the four configurations are shown in Fig. 5(a). Recall that this rate corresponds to the damping of flows in the approximate direction of symmetry. The QHS and 2\% Hill are very similar in this quantity, except for the increase in the damping in the vicinity of the $t=12 / 11$ magnetic island. The increase in the vicinity of the island is by a factor of between 4 and 5 . The island has a width of $3 \mathrm{~mm}$ effective minor radius out of a total effective minor radius of $11.3 \mathrm{~cm}$, and the increased damping dies away within $0.5 \mathrm{~mm}$ on either side of the magnetic island chain. Furthermore, the fraction of the total volume enclosed by these islands is only $\approx 6 \%$ of the volume within the 
LCMS. We thus infer that the effects of this small island chain are not significant.

The damping due to this island in the QHS configuration can be compared to the large increase in damping across the entire minor radius in the $14 \%$ Mirror configuration. This configuration has a large $t=8 / 7$ island, whose effect is visible as only a factor of 2 perturbation to the already large slow damping rate. Once again, it is inferred that the effect of this island will be small.

The most impressive island induced change in the slow damping rate is that in the $6 \%$ Hill configuration. On surfaces near the magnetic island, the increase in the viscous damping is quite large. On the other hand, both Hill configurations and the QHS configuration have comparable slow damping rates at the edge and near the center, where the impact of magnetic island distortions are minimal.

The fast damping rates are shown in Fig. 5(b). Recall that this rate corresponds to the damping of flows across the direction of symmetry. Away from the islands, all four configurations have similar fast damping rates to within a factor of 2. In the vicinity of the magnetic islands, the increase in the fast flow damping rate is quite large in all three configurations, which contain an island chain. This increase in the fast rate due to the islands is apparently the dominant feature on the graph, and can overwhelm the differences in the fast rate due to, for instance, the addition of the $(n, m)=(4,0)$ symmetry breaking components in the $14 \%$ Mirror configuration.

Given the resonant denominators in the viscosity coefficients [see Eqs. (5)], it might appear that the small values of $|n-m t|$ near the rational surfaces might increase the calculated the viscous damping. We have found that this is not the case for the calculations presented above, because the $b_{n, m}$ associated with the resonances are also very small. Hence, the increase in viscous damping is due to the increase in certain spectral components associate with deformed surface shape, not a precipitous decrease in the denominators.

\section{CONCLUSIONS}

Four configurations of the HSX stellarator have been considered from the standpoint of the viscous damping of plasma flows due to magnetic islands. The interaction of the main helical spectral component and the island perturbation produce new spectral components that break the quasisymmetry. The base quasihelically symmetric configuration has a $t=12 / 11$ island chain, which contributes a small but not problematic increase in viscous damping on surfaces very near the island. The viscous damping can be raised compared to the quasisymmetric configuration, either by the introduction of a large $(n, m)=(4,0)$ symmetry breaking spectral component or through the introduction of large islands like those present when the $t=4 / 4$ resonant surface is present inside the LCFS.

\section{ACKNOWLEDGMENTS}

The authors would like to thank John Canik for help with the Biot-Savart code which was used in the calculations presented here and all the members of the HSX lab for generously allocating the computing resources which made these calculations possible. They also wish to thank Chris Hegna for helpful discussions.

This work was funded by the U.S. Department of Energy.

${ }^{1}$ F. S. B. Anderson, A. F. Almagri, D. T. Anderson, P. G. Mathews, J. N. Talmadge, and J. L. Shohet, Fusion Technol. 27, 273 (1995).

${ }^{2}$ J. Nuhrenberg and R. Zille, Phys. Lett. A 129, 113 (1988).

${ }^{3}$ A. A. Galeev, R. Z. Sagdeev, H. P. Furth, and M. N. Rosenbluth, Phys. Rev. Lett. 22, 511 (1969).

${ }^{4}$ K. C. Shaing and J. D. Callen, Phys. Fluids 26, 3315 (1983).

${ }^{5}$ K. C. Shaing, Phys. Rev. Lett. 87, 245003 (2001).

${ }^{6}$ S. R. Hudson, D. A. Monticello, A. H. Reiman, A. H. Boozer, D. J. Strickler, S. P. Hirshman, and M. C. Zarnstorff, Phys. Rev. Lett. 89, 275003 (2002).

${ }^{7}$ M. C. Zarnstorff, L. A. Berry, A. Brooks, E. Fredrickson, G.-Y. Fu, S. Hirshman, S. Hudson, L.-P. Ku, E. Lazarus, D. Mikkelsen, D. Monticello, G. H. Neilson, N. Pomphrey, A. Reiman, D. Spong, D. Strickler, A. Boozer, W. A. Cooper, R. Goldston, R. Hatcher, M. Isaev, C. Kessel, J. Lewandowski, J. F. Lyon, P. Merkel, H. Mynick, B. E. Nelson, C. Nuehrenberg, M. Redi, W. Reiersen, P. Rutherford, R. Sanchez, J. Schmidt, and R. B. White, Plasma Phys. Controlled Fusion 43, A237 (2001).

${ }^{8}$ F. Wagner, J. Baldzuhn, R. Brakel, R. Burhenn, V. Erckmann, T. Estrada, P. Grigull, H. J. Hartfuss, G. Herre, M. Hirsch, J. V. Hofmann, J. Jaenicke, A. Rudyj, U. Stroth, and A. Weller, Plasma Phys. Controlled Fusion 36, A61 (1994).

${ }^{9}$ M. Hirsh, P. Grigull, H. Wobig, J. Kisslinger, K. McCormick, M. Manton, J. Baldzuhn, S. Fiedler, C. Fuchs, J. Geiger, L. Giannone, H. J. Hartfuss, E. Holzhauer, R. Jaenicke, M. Kick, H. Maassberg, F. Wagner, and A. Weller, Plasma Phys. Controlled Fusion 42, A231 (2000).

${ }^{10} \mathrm{H}$. Wobig and J. Kisslinger, Plasma Phys. Controlled Fusion 42, 823 (2000).

${ }^{11}$ K. H. Burrell, Phys. Plasmas 4, 1499 (1997).

${ }^{12}$ F. Sano, T. Mizuuchi, K. Nagasaki, H. Okada, S. Kobayashi, K. Kondo, K. Hanatani, Y. Nakamura, M. Nakasuga, S. Besshou, S. Yamamoto, Y. Suzuki, Y. Manabe, H. Shidara, T. Takamiya, Y. Ohno, Y. Nishioka, H. Kukimoto, K. Takahashi, Y. Fukagawa, H. Kawazome, H. Kaneko, S. Tsuboi, S. Nakazawa, S. Nishio, M. Yamada, Y. Ijiri, T. Senju, K. Yaguchi, K. Sakamoto, K. Tohshi, M. Shibano, V. Tribaldos, F. Tabares, and T. Obiki, Proceedings of the 14th Stellarator Workshop, Greifswald, Germany, 2003.

${ }^{13}$ K. Ida, S. Inagaki, N. Tamura, T. Morisaki, N. Ohyabu, K. Khlopenkov, S. Sudo, K. Watanabe, M. Yokoyama, T. Shimazuma, Y. Takeiri, K. Itoh, M. Yoshinuma, Y. Liang, K. Narihara, K. Tanaka, Y. Nagayama, T. Tokuzawa, K. Kawahata, H. Suzuki, A. Komori, T. Akiyama, N. Ashikawa, M. Emoto, H. Funaba, P. Goncharov, M. Goto, H. Idei, K. Ikeda, M. Isobe, O. Kaneko, H. Kawazome, T. Kobuchi, A. Kostriukov, S. Kubo, R. Kumazawa, S. Masuzaki, T. Minami, J. Miyazawa, S. Morita, S. Murakami, S. Muto, T. Mutoh, Y. Nakamura, H. Nakanishi, Y. Narushima, K. Nishimura, N. Noda, T. Notake, H. Nozato, S. Ohdachi, Y. Oka, M. Osakabe, T. Ozaki, B. J. Peterson, A. Sagara, T. Saida, K. Saito, S. Sakakibara, R. Sakamoto, M. Sasao, K. Sato, M. Sato, T. Seki, M. Shoji, N. Takeuchi, K. Toi, Y. Torii, K. Tsumori, T. Watari, Y. Xu, H. Yamada, S. Yamamoto, T. Yamamoto, Y. Yoshimura, I. Ohtake, K. Ohkubo, T. Mito, T. Satow, T. Uda, K. Yamazaki, K. Matsuoka, O. Motojima, and M. Fujiwara, Nucl. Fusion 44, 290 (2004).

${ }^{14}$ M. Coronado and J. N. Talmadge, Phys. Fluids B 5, 1200 (1993).

${ }^{15}$ K. C. Shaing and E. C. Crume, Jr., Phys. Rev. Lett. 63, 2369 (1989).

${ }^{16}$ W. D. D'haeseleer, W. N. G. Hitchon, J. D. Callen, and J. L. Shohet, Flux Coordinates and Magnetic Field Structure (Springer, Berlin, 1991).

${ }^{17}$ S. P. Gerhardt, Ph.D. dissertation, University of Wisconsin-Madison, 2004.

${ }^{18}$ G. Kuo-Petravic, A. H. Boozer, J. A. Rome, and R. H. Fowler, J. Comput. Phys. 51, 261 (1983).

${ }^{19}$ K. C. Shaing, S. P. Hirshman, and J. D. Callen, Phys. Fluids 29, 521 (1994); M. Coronado and H. Wobig, ibid. 29, 527 (1994).

${ }^{20}$ S. P. Hirshman, Nucl. Fusion 18, 917 (1978).

${ }^{21}$ K. Narihara, K. Y. Watanabe, I. Yamada, T. Morisaki, K. Tanaka, S. Sakakibara, K. Ida, R. Sakamoto, N. Ohyabu, N. Ashikawa, M. Ernoto, H. Funaba, M. Goto, H. Hayashi, H. Idei, K. Ikeda, S. Inagaki, N. Inoue, O. Kaneko, K. Kawahata, T. Kobuchi, A. Komori, S. Kubo, R. Kumazawa, S. 
Masuzaki, J. Miyazawa, S. Morita, O. Motojima, S. Murakami, S. Muto, T. Mutoh, Y. Nagayama, Y. Nakamura, H. Nakanishs, K. Nishimura, N. Noda, T. Notake, S. Ohdachi, Y. Oka, K. Okhubo, M. Osakabe, S. Ozaki, B. J. Peterson, A. Sagara, K. Saito, H. Sasao, M. Sasao, K. Sato, M. Sato, T. Seki, T. Shimozuma, C. Shoji, S. Sudo, H. Suzuki, A. Takayama, M.
Takechi, Y. Takeiri, K. Toi, N. Tokuzawa, Y. Torii, K. Tsumori, T. Watari, H. Yamada, S. Yamaguchi, S. Yamamoto, K. Yamazaki, and Y. Yoshimura, Phys. Rev. Lett. 87, 135002 (2001).

${ }^{22}$ A. Bhattacharjee, T. Hayashi, C. C. Hegna, N. Nakajima, and T. Sato, Phys. Plasmas 2, 883 (1995). 